\title{
The Erlangen test of activities of daily living in persons with mild dementia or mild cognitive impairment (ETAM) - an extended validation
}

\author{
Stephanie Book ${ }^{1 *}$ D, Katharina Luttenberger ${ }^{1}$, Mark Stemmler ${ }^{2}$, Sebastian Meyer ${ }^{3}$ and Elmar Graessel ${ }^{1}$
}

\begin{abstract}
Background: The ability to perform activities of daily living (ADLs) is a central marker in the diagnosis and progression of the dementia syndrome. ADLs can be identified as basic ADLs (BADLs), which are fairly easy to perform, or instrumental ADLs (IADLs), which involve more complex activities. Presently, the only performance-based assessment of IADL capabilities in persons with cognitive impairment is the Erlangen Test of Activities of Daily Living in Persons with Mild Dementia or Mild Cognitive Impairment (ETAM). The aim of the present study was to revalidate the ETAM in persons with mild cognitive impairment $(\mathrm{MCl})$ or mild dementia and to analyze its application to persons with moderate dementia.
\end{abstract}

Methods: We used baseline data from a cluster randomized controlled trial involving a sample of 443 users of 34 day-care centers in Germany. We analyzed groups of persons with $\mathrm{MCl}$, mild dementia, and moderate dementia, categorized on the basis of the Mini-Mental State Examination (MMSE) and the Montreal Cognitive Assessment (MoCA). An item analysis was performed, and new discriminant validities were calculated. We computed a confirmatory factor analysis (CFA) to examine the postulated theoretical model of the ETAM with all six items loading on a single IADL factor. This was the first time that the ETAM's sensitivity to change was analyzed after a time period of 6 months.

Results: The overall sample scored on average 17.3 points ( $S D=7.2$ ) on the ETAM (range: 0-30 points). Persons with $\mathrm{MCl}$ scored on average 23.2 points, persons with mild dementia scored 18.4 points, and persons with moderate dementia scored 12.9 points, $p<.001$ (ANOVA). The item analysis yielded good difficulty indices and discrimination powers. The CFA indicated a good fit between the model and the observed data. After 6 months, both the ETAM score at baseline and the change in MMSE score (t0-t 1 ) were significant predictors of the ETAM score at $\mathrm{t} 1$.

Conclusions: The ETAM is a valid and reliable instrument for assessing IADL capabilities in persons with MCI or mild dementia. It is sensitive to changes in cognitive abilities. The test parameters confirm its application to persons with moderate dementia.

Trial registration: Identifier: ISRCTN16412551 (Registration date: 30 July 2014, registered retrospectively).

Keywords: Activities of daily living, Cognitive impairment, Dementia, Performance test, Validation

\footnotetext{
* Correspondence: stephanie.book@uk-erlangen.de

${ }^{1}$ Center for Health Services Research in Medicine, Department of Psychiatry

and Psychotherapy, Friedrich-Alexander Universität Erlangen-Nürnberg,

Schwabachanlage 6, 91054 Erlangen, Germany

Full list of author information is available at the end of the article
}

(c) The Author(s). 2018 Open Access This article is distributed under the terms of the Creative Commons Attribution 4.0 International License (http://creativecommons.org/licenses/by/4.0/), which permits unrestricted use, distribution, and

reproduction in any medium, provided you give appropriate credit to the original author(s) and the source, provide a link to the Creative Commons license, and indicate if changes were made. The Creative Commons Public Domain Dedication waiver (http://creativecommons.org/publicdomain/zero/1.0/) applies to the data made available in this article, unless otherwise stated. 


\section{Background}

The mastery of everyday practical capacities is essential for the elderly to maintain their independence. Lawton and Brody [1] defined a set of everyday activities for the elderly, so-called activities of daily living (ADLs). They differentiated between basic ADLs (BADLs), which refer to self-maintenance skills such as feeding, dressing, and toileting, and instrumental ADLs (IADLs), which cover more complex behaviors of domestic functioning and enable independent living. IADLs include food/meal preparation, financial administration, housekeeping, laundry, use of the telephone, responsibility for one's own medication, mode of transportation, and shopping.

With an aging population, the number of people with dementia has dramatically increased in recent decades, and dementia has become a public health challenge [2]. Alzheimer's disease is the most common form of dementia and begins years before the onset of clinical symptoms. Its pathology can be described on a continuum that ranges from a preclinical stage (changes in biomarkers) to a prodromal stage with minor cognitive symptoms/mild cognitive impairment, to a symptomatic stage that includes dementia [3, 4]. At different stages of the disease, different assessments are needed. While a patient is in the preclinical stage, an assessment of biomarkers is most important, whereas functional assessments become more important in the prodromal and symptomatic stages [5]. IADLs can be used for a functional assessment as early as in the prodromal stage because it has been shown that impairments in IADLs are associated with the diagnosis and development of dementia [6-10] and, more important, deficits in BADLs and IADLs seem to occur at different stages of the dementing process $[10,11]$. Whereas BADLs have been found to be more strongly correlated with motor functioning and coordination [12] and thus are more likely to remain preserved until the later stages of the disease, IADLs have been found to be more sensitive to the earlier stages of cognitive decline as these activities are more complex and require greater neuropsychological organization [11]. Even more, IADL impairments have been shown to predict the progression to dementia and can be used to help distinguish between dementia and early forms of cognitive decline, such as mild cognitive impairment (MCI) $[6,13]$. MCI refers to a state that is defined by the presence of the first cognitive impairments that do not yet constitute dementia [14] but have a high probability of progressing to dementia [15]. Persons with MCI can experience subtle changes in everyday functional competence [8]. There is scientific evidence showing that IADLs can be impaired in MCI [8, 16-18]. In addition, in a systematic review, Jekel et al. [17] reported that patients with MCI and IADL deficits seem to have a higher risk of developing dementia than patients with MCI without IADL deficits, again stressing the importance of IADLs.

Because there is ample evidence that the ability to perform IADLs plays a crucial role in identifying the development of the dementia syndrome, there is a need for assessment tools that have been specifically designed and validated for patients with the first signs of impairments in IADLs (i.e. persons with MCI or mild dementia). As one study showed that several informant-based IADL questionnaires were limited in their quality [19], it remains important to identify an optimal way to measure IADLs. A promising approach is the use of performance tests as these tests provide standardized and more objective results [17]. To move in this conceptual direction, the Erlangen Test for Activities of Daily Living (E-ADL) [20] was developed in 2009 and can be characterized by its excellent economy. In contrast to other performance tests, it requires only about $10 \mathrm{~min}$ to be performed and does not require any tasks to be done outside the test room. The E-ADL was designed to assess BADL capabilities and can be used with persons with moderate or severe dementia [21]. Because it is too easy for persons with less severe dementia, there is a need for a performance test that has been validated for persons with mild dementia or even MCI. For this reason, the Erlangen Test of Activities of Daily Living in Persons with Mild Dementia or Mild Cognitive Impairment (ETAM) was developed as a performance-based tool for the assessment of IADLs [22]. The ETAM addresses some of the disadvantages of existing performance tests for ADL capabilities as some of these are very time-consuming (from $45 \mathrm{~min}$, Functional Living Skills Assessment [FLSA] [23], up to $1.5 \mathrm{~h}$, Direct Assessment of Functional Abilities [DAFA] [24]), cover only a limited range of relevant domains of IADLs, or include culture-specific items (e.g. "calling directory assistance" or "refilling a prescription" in the Revised Direct Assessment of Functional Status [DAFS-R] [25]). Above all, the ETAM can be used with persons with MCI [22]. In a first validation study of 107 study participants, including participants with normal cognition, persons with $\mathrm{MCI}$, and persons with mild dementia, the ETAM was shown to be a feasible performance-based assessment tool with good psychometric parameters [22]. In this first study, the final structure of the ETAM was developed, and the items were reduced from ten to six items on the basis of an exploratory factor analysis and other criteria.

However, because this study was only cross-sectional, there is currently no longitudinal data on the ETAM's sensitivity to change. This is essential because sensitivity to change or responsiveness is an essential aspect of validity. It provides important information about the ETAM's ability to measure change over time, and consequently, it determines whether the ETAM can be used in intervention studies. At this time, there are currently 
no performance tests for assessing IADLs in persons with MCI that can be used in intervention studies. Thus, one aim of the present study was to analyze the ETAM's sensitivity to change. In addition, we wanted to investigate whether the original target group of persons with MCI or mild dementia could also be extended to include persons with moderate dementia. This would extend the application of the ETAM enormously because dementia is a progressive disease. Other aims of the present study involve other test construction criteria. The exploratory factor analysis in the validation study supported a one-factor structure for the ETAM. In the current study, we conducted a confirmatory factor analysis and investigated whether this structure could be supported. This was important to do in order to determine whether actual data were consistent with the hypothesis that the ETAM consists of a single IADL factor. Other test construction criteria included analyzing discriminant validity with additional instruments and determining criterion-related validity.

\section{Methods \\ Design}

The data for the extended validation were obtained from the two-arm cluster randomized controlled trial "DeTaMAKS project" (ISRCTN16412551) to evaluate a six-month-long multimodal non-pharmacological therapy (MAKS therapy) in day-care centers in Germany with day-care-center users and their caregivers. The study protocol was published previously [26]. For the current study, we included baseline data (t0) from all day-care-center users and follow-up data after six months (t1) for the control group that received no study-specific treatment (Fig. 1). The MAKS intervention is a multimodal nonpharmacological therapy for older adults with mild to moderate dementia and has been shown to be an effective treatment for dementia [27]. Because of the influence of the MAKS therapy on the ETAM scores [28], all analyses with t1 data were computed only on data from the control group, which did not receive any special therapy during that time. Cross-sectional analyses from the first measurement point $(\mathrm{t} 0)$ were computed on data from all participants (the later control and the later intervention groups).

All procedures were approved by the Friedrich-Alexander-Universität Erlangen-Nürnberg Ethics Committee (Re.-No. 170_14 B).

\section{Recruitment}

All users of the 34 day-care centers throughout Germany and their caregivers were included in the screening process. All dyads (consisting of a day-care center user and caregiver) that fulfilled the criteria for inclusion were informed about the study and asked to take part in the project. Exclusion criteria for the day-care-center users were: blindness, deafness, lacking a caregiver, lacking the ability to communicate, more than one stroke, severe depression, schizophrenia, an addictive disorder, concrete plans for institutionalization, and attendance at the day-care center of less than once a week. The day-care centers' documentation contained all medical diagnoses and doctors' prescriptions known to the informal caregivers. Inclusion criteria were informed consent and an MMSE score of 10 or higher. For persons with an MMSE score of 24 or higher, we required them to also have an MoCA score of 22 or lower. Recruitment strategies are described in detail in [26].

\section{Instruments}

\section{Tool under investigation}

Erlangen Test of Activities of Daily Living in Persons with Mild Dementia or Mild Cognitive Impairment (ETAM) [22] The ETAM is a feasible (19 min on average), reliable, and valid performance test for IADL capabilities in persons with MCI or mild dementia. Thus, it can be administered to investigate the capacity to accomplish complex activities of daily living relevant to older adults living alone. The development of the ETAM was theoretically driven by the International Classification of Functioning, Disability, and Health (ICF), which was published by the World Health Organization (WHO) in 2001 [29]. The ICF is a classification of health and health-related domains including a list of "Body Functions," "Body Structures," "Activity and Participation," and "Environmental Factors." Activity can be described as the "execution of a task or action by an individual" (p. 123) and Participation can be described as "involvement in a life situation" (p. 123). The domain "Activity and Participation" consists of nine main categories, five of which are particularly relevant for the independent living of persons with dementia [30]: Communication, Mobility, Self-Care, Domestic Life, and Major Life Areas, especially Economic Life. These main categories are represented by six items in the ETAM (one 6-point item for each main category with the exception of two 3-point items for the main category Domestic Life). The total possible score is 30 points with higher values indicating greater competence in the mastery of IADLs. In a first validation study [22], Cronbach's alpha was .71, and the inter-rater reliability was .97 .

\section{Control tools}

Mini-Mental State Examination (MMSE) [31] The MMSE is the most frequently used short screening instrument for dementia [32]. It assesses five areas of cognitive function: orientation, registration, attention and calculation, recall, and language. Designed to be a short (5-10 min) pencil-and-paper test that is easy to administer, it is based 


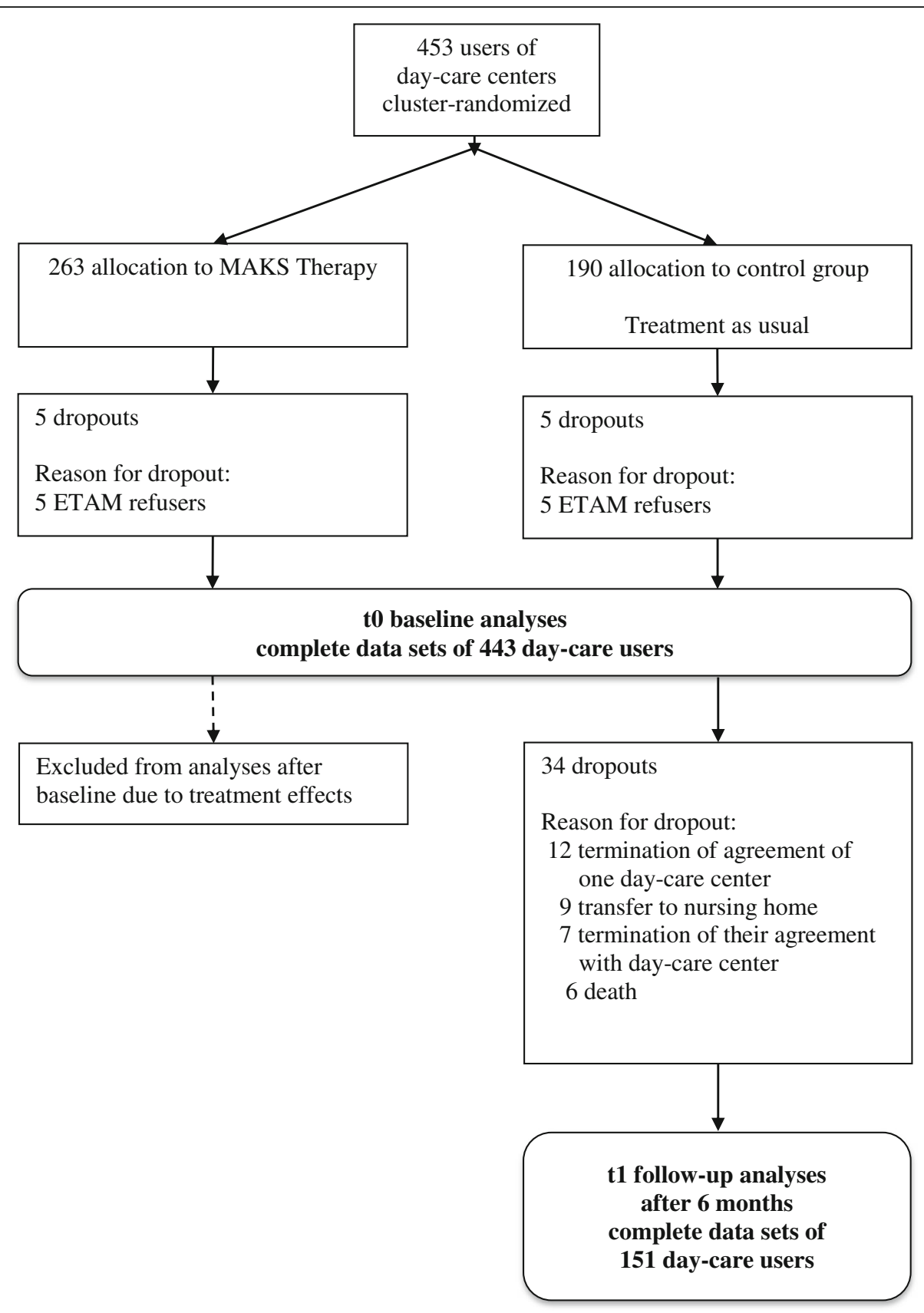

Fig. 1 Consort flow chart

on a total possible score of 30 points, with higher values indicating greater performance capacity. Scores $\geq 24$ points are considered to be indicative of "normal" cognition (not associated with dementia), whereas scores below this can be indicative of mild dementia (18-23 points), moderate dementia (10-17 points), or severe dementia (0-9 points) [33].

Montreal Cognitive Assessment (MoCA) [34] The $\mathrm{MoCA}$ is a measure that is used to screen for MCI. It consists of more difficult items than the MMSE and is thus able to better detect MCI [34-37]. Scores range from 0 to 30 points, with higher scores indicating better cognitive performance. A score of 22 or lower indicates cognitive impairment [35].

EuroQol five dimensions questionnaire (EQ-5D) [38] The EQ-5D is a cognitively simple, brief instrument providing a simple description of a person's generic health status. It consists of five items covering Mobility, 
Self-Care, Usual Activities, Pain/Discomfort, and Anxiety/Depression. Each item is rated on a 5-point scale indicating the level of severity with higher scores reflecting more complaints.

Nurses' Observation Scale for Geriatric Patients (NOSGER) [39] The NOSGER is an observer rating scale covering the impairments that are found most frequently in geriatric patients. It consists of six subscales (Mood, Disturbing Behavior, Social Behavior, Memory, ADLs, and IADLs) that contain five items each. We included the Social Behavior subscale in the present study. Each item is rated on a scale that ranges from 1 (always) to 5 (never) with higher scores indicating less impairment.

\section{Other measures}

Each participant's age, gender, nursing care needs, and other sociodemographic data were provided by their caregivers or nurses at the day-care center. In Germany, nursing care needs are determined on the basis of a three-level scale to establish eligibility for nursing care benefits. Three care levels describe the extent to which the patient is eligible to receive assistance from longterm care insurance ranging from mild care (level 1) to moderate care (level 2) to a great need for care (level 3).

\section{Classification of the level of cognitive impairment (MCl, mild or moderate dementia)}

In order to differentiate between day-care-center users with $\mathrm{MCI}$, mild dementia, or moderate dementia, we administered a combination of the MMSE and the MoCA at baseline. The MoCA was administered when the MMSE values ranged from 24 to 30 points, as the MMSE is widely regarded as not being sensitive enough to be able to detect $\mathrm{MCI}$ in the range of non-dementia cases [33, 34, 40]. Freitas [35] suggested using a cut-off score of 22 points to discriminate between normal cognition and MCI for the MoCA. To differentiate between mild and moderate dementia, we used the MMSE values and the recommendations by Tombaugh et al. [33]. We considered scores between 18 and 23 points to be indicative of mild dementia and scores between 10 and 17 points to be indicative of moderate dementia. With this procedure, we defined the level of cognitive impairment in a psychometric way.

\section{Data recording}

The MMSE, MoCA, ETAM, and anamnestic data were recorded by staff at the day-care centers who had attended training sessions. The EQ-5D and the NOSGER subscale Social Behavior were completed via computer-assisted telephone interviews (CATIs) with the caregivers. All of the persons involved in data recording were thoroughly trained in the use of each instrument.

\section{Data quality management}

In order to ensure the validity of the data, the data sources (tests, CATIs, day-care centers) are subjected to a random internal audit. To obtain evidence of the inter-rater reliability of the ETAM test and the CATI, $5 \%$ of the baseline data were collected with the participation of a second person who was there to observe. For additional information, please see [28].

\section{Sample}

For the purpose of this study, our analyses were based on 443 day-care-center users with baseline data. The proportion of women in the sample was $61.4 \%$, and the mean age was 81.7 years $(\mathrm{SD}=7.7)$. All analyses except for one were based on these 443 day-care-center users and their baseline data (Table 1). Only for the analysis of sensitivity to change did we use the baseline and follow-up data of 151 control-group participants (because of treatment effects, we excluded the intervention group, see Fig. 1).

\section{Statistical analysis \\ Reliability and item analysis}

In order to determine the test construction characteristics of the ETAM, measures of reliability were computed

Table 1 Sample characteristics

\begin{tabular}{ll}
\hline Characteristics & $N=443$ \\
\hline Age, M (SD) & $81.7(7.7)$ \\
Women, $n$ (\%) & $272(61.4 \%)$ \\
Education, $n$ (\%) & \\
$\quad$ Not completed & $24(5.4 \%)$ \\
9 years & $317(71.6 \%)$ \\
10 years & $51(11.5 \%)$ \\
13 years & $23(5.2 \%)$ \\
More than 13 years & $28(6.3 \%)$ \\
Care level & \\
No care level & $71(16 \%)$ \\
Level 1 & $232(52.4 \%)$ \\
Level 2 & $136(30.7 \%)$ \\
Level 3 & $4(0.9 \%)$ \\
MMSE score, M (SD) & $19.4(4.7)$ \\
Cognitive Impairment according to MMSE/MoCA & \\
MCI (MMSE 24-30 and MoCA $\leq 22)$ & $91(20.5 \%)$ \\
Mild dementia (MMSE 18-23) & $186(42.0 \%)$ \\
Moderate dementia (MMSE 10-17) & $166(37.5 \%)$ \\
ETAM score at baseline, M (SD) & $17.3(7.2)$ \\
\hline MMSE mini-mental status examination, ETAM Erlangen test of activities of daily \\
living in persons with mild dementia or mild cognitive impairment
\end{tabular}


and an item analysis was conducted. Means and standard deviations were calculated at the item level and for the ETAM total score. Cronbach's $\alpha$ was computed as a measure of internal consistency, and values higher than 0.7 were considered acceptable [41]. The difficulty index and discrimination power were calculated at the item level. As the ETAM items use a multilevel (4- or 7-step) response format, the difficulty index was calculated as the ratio of the subjects' squared points to the number of subjects times the squared item maximum $\left(\frac{\sum_{i=1}^{n} x_{i}^{2}}{n \cdot x_{\max }^{2}}\right)$ [42]. The item difficulty index ranges from 0 (most difficult item) to 1 (easiest item). Item difficulties in the range of .2 to .8 are preferred [43]. Discrimination power was calculated as the corrected item-total correlation. A discrimination power of .3 to .5 should be rated as moderate, whereas a discrimination power $>.5$ should be rated as high [43].

In order to assess the extent to which the ETAM could discriminate between different levels of severity of cognitive impairment, we computed a one-way ANOVA with the total ETAM score as the dependent variable and the severity of cognitive impairment (MCI, mild dementia, moderate dementia) as the independent variable. For a post hoc analysis, we computed a Games-Howell test because the groups did not have equal variances. Cohen's $d$ was used to examine the magnitude of the differences in ETAM scores between the subgroups ( $\mathrm{MCI}$, mild dementia, moderate dementia).

\section{Confirmatory factor analysis}

A confirmatory factor analysis was computed to determine whether the six ETAM items fit the proposed one-factor model as found when the exploratory factor analysis was conducted in the first validation study [22]. The asymptotic distribution free (ADF) method of estimation was chosen because the ETAM items were not normally distributed.

To evaluate the model, we used the adjusted chi-square test statistic in conjunction with other fit indices as recommended by Brown [44]. Schreiber et al. [45] recommended the ratio of $x^{2}$ to $\mathrm{df} \leq 2$, a root mean square error of approximation (RMSEA) $<.06$, a comparative fit index $(\mathrm{CFI}) \geq .95$, and a Tucker-Lewis index $(\mathrm{TLI}) \geq .96$.

\section{Validity}

Discriminant validity To test for discriminant validity, the ETAM score at baseline was correlated with several scales or items that measured different constructs (Spearman Rank Sum Correlation) at baseline. The MMSE was used to measure cognition, the Social Behavior scale of the NOSGER was used to measure social behavior, and the five items of the EQ-5D were used to measure specific topics that are relevant for health status: Mobility, Self-Care, Usual Activities, Pain/Discomfort, and Anxiety/Depression. We hypothesized that the correlation between the ETAM and the MMSE would be around .5 because the two tests measure the progression of the same disease, whereas the correlations between the ETAM and the other tests were expected to be low (.2 or lower).

Criterion-related validity The variable nursing care needs is an appropriate independent external criterion that was used to assess criterion-related validity. It is determined by external raters working for the "Medical Service of Health Insurances" and is a health measure with relevance to a person's economic standing because it determines the amount of access a person has to financial assistance. We wanted to test the hypothesis that participants achieve significantly different ETAM scores depending on their care level. We computed a one-way ANOVA with the ETAM score at baseline as the dependent variable and nursing care needs as the independent variable. For a post hoc analysis, we computed Hochberg's GT2 because the population variances were equal but the sample sizes were very different.

Sensitivity to change: Subgroup analysis of the control group We wanted to assess the ETAM's sensitivity to reflect change in cognitive abilities that occurred over six months in the subgroup of participants in the control group $(n=151)$ from the "DeTaMAKS" project. We expected that participants with larger decreases in their cognitive abilities as measured with the MMSE would also show larger decreases in their IADL capacities as measured with the ETAM. A regression analysis was computed with the ETAM score at follow-up (t1) as the criterion and the ETAM score at baseline (t0) and the MMSE change score from t0 to $\mathrm{t} 1$ as predictors.

The analyses were computed on the baseline data for the sample consisting of participants with MCI, mild dementia, or moderate dementia $(N=443)$ and for the subgroups with MCI (MMSE score 24-30 and MoCA $\leq 22$ ), mild dementia (MMSE score 18-23), and moderate dementia (MMSE score 10-17). For reasons of comparability, we also report results for the original group that was targeted by the ETAM (i.e. persons with MCI or mild dementia; $n=277$ ) when similar analyses were carried out in the first validation study [22].

IBM SPSS Statistics 21 was used for most of the statistical analyses. Stata 13.1 was used for the confirmatory factor analysis. 


\section{Results}

\section{Reliability and item analysis}

For the total sample consisting of participants with MCI, mild dementia, or moderate dementia $(N=443)$, the mean ETAM score was 17.3 points with a standard deviation of 7.2. The median was 18.0 points. The distribution had a skewness of -.264 and a kurtosis of -.852 . The maximum range of 0 to 30 points was completely covered. Cronbach's alpha was .79. For the group comparisons, the following Cohen's d values were found: $d=0.84$ for MCI $(n=91)$ versus mild dementia $(n=186), \mathrm{d}=1.70$ for $\mathrm{MCI}$ versus moderate dementia $(n=166)$, and $\mathrm{d}=0.85$ for mild versus moderate dementia. For the original target group of participants with $\mathrm{MCI}$ or mild dementia $(n=277)$, the mean ETAM score was 20.0 points $(S D=6.2)$ with a Cronbach's alpha of .74. The mean ETAM scores differed significantly between $\mathrm{MCI}$, mild dementia, and moderate dementia, $\mathrm{F}(2$, $440)=87.85, p<.001$. A post hoc Games-Howell test revealed that all ETAM scores differed significantly from each other at $\mathrm{p}<.001$ : Participants with MCI $(\mathrm{n}=91)$ scored on average 23.2 points (95\% CI 22.2-24.2), participants with mild dementia $(\mathrm{n}=186)$ scored 18.4 points (95\% CI 17.5-19.3), and participants with moderate dementia $(n=166)$ scored 12.9 points $(95 \% \mathrm{CI}$ 11.9-14.0); see Fig. 2.

The overall discriminatory powers were high, ranging from .49 to .64 . Only in the subgroup of persons with MCI did the item "alarm clock" have a low discriminatory power of .25 . Other items in this subgroup had moderate discriminatory powers ranging from .35 to .44 . In the subgroup of persons with mild dementia, the discriminatory power ranged from .45 to .56 , and in the subgroup of persons with moderate dementia, from .39 to .63. Overall, the most difficult item was "phone call" (.25), whereas "making tea" (.67) was the easiest item. Again, only for the subgroup of persons with MCI, the easiest items were "pill organizer" and "alarm clock" (both .83) for which the difficulties ranged from .44 to .83. In the subgroup of persons with mild dementia, the difficulties ranged from .25 to .71. In the subgroup of persons with moderate dementia, the difficulties ranged from .13 to .56. Item characteristics are presented in Table 2.

\section{Confirmatory factor analysis}

We hypothesized a one-factor model. For the extended total sample of persons with MCI, mild dementia, or moderate dementia, the model indicated a good fit to the data, $X^{2}(9, N=443), p=.088$. The ratio of $\chi^{2}$ to $\mathrm{df}$ was 1.68 . The CFI was .975, the TLI was .959, and the RMSEA was .039. Similar results were found for the original target group of participants with MCI or mild dementia: $X^{2}(9, n=277), p=.359$. The ratio of $\chi^{2}$ to $\mathrm{df}$ was 1.10 . The CFI was .991, the TLI was .985 , and the RMSEA was .019. These values indicate a good fit between the model and the observed data.

\section{Validity \\ Discriminant validity}

Overall, the correlation of the ETAM total score with the MMSE was .59; for the subgroups, the correlations were .20 for MCI, .24 for mild dementia, and .40 for moderate dementia. For the original target group of participants with MCI or mild dementia, the correlation with the MMSE was .43. The ETAM total score was hardly correlated with the other items of the EQ-5D:

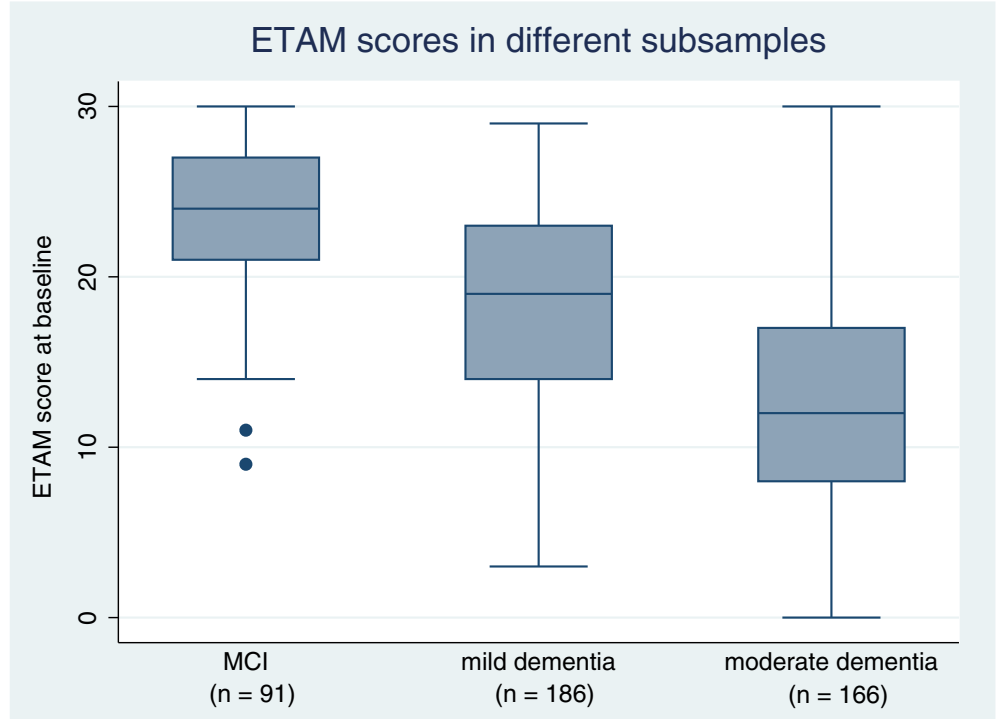

Fig. 2 Boxplots of the ETAM scores in persons with $\mathrm{MCl}$, mild dementia, or moderate dementia 
Table 2 Item characteristics of the ETAM

\begin{tabular}{|c|c|c|c|c|c|c|c|}
\hline Characteristics & Making tea & Alarm clock & Pill organizer & Finances & Traffic situations & Phone call & ETAM total score \\
\hline Score Range & $0-3$ & $0-3$ & $0-6$ & $0-6$ & $0-6$ & $0-6$ & $0-30$ \\
\hline \multicolumn{8}{|l|}{ Mean (SD) } \\
\hline Total & $2.3(1.0)$ & $2.2(0.9)$ & $3.7(2.3)$ & $3.6(2.0)$ & $3.3(1.9)$ & $2.4(1.8)$ & $17.3(7.2)$ \\
\hline $\mathrm{MCl}$ & $2.5(0.8)$ & $2.7(0.6)$ & $5.3(1.4)$ & $5.0(1.5)$ & $4.2(1.6)$ & $3.6(1.7)$ & $23.2(4.7)$ \\
\hline Mild dementia & $2.4(0.9)$ & $2.3(0.9)$ & $4.0(2.1)$ & $3.7(1.9)$ & $3.5(1.7)$ & $2.5(1.7)$ & $18.4(6.2)$ \\
\hline Moderate dementia & $2.0(1.1)$ & $1.7(1.0)$ & $2.5(2.3)$ & $2.7(2.0)$ & $2.5(1.9)$ & $1.6(1.5)$ & $12.9(6.7)$ \\
\hline \multicolumn{8}{|l|}{ Discriminatory power } \\
\hline Total & .49 & .53 & .59 & .61 & .54 & .64 & \\
\hline $\mathrm{MCl}$ & .37 & .25 & .35 & .44 & .42 & .40 & \\
\hline Mild dementia & .50 & .45 & .46 & .46 & .51 & .56 & \\
\hline Moderate dementia & .45 & .42 & .50 & .59 & .39 & .63 & \\
\hline \multicolumn{8}{|l|}{ Difficulty } \\
\hline Total & .67 & .61 & .53 & .47 & .39 & .25 & \\
\hline $\mathrm{MCl}$ & .77 & .83 & .83 & .44 & .57 & .44 & \\
\hline Mild dementia & .71 & .66 & .58 & .48 & .42 & .25 & \\
\hline Moderate dementia & .56 & .43 & .31 & .31 & .27 & .13 & \\
\hline \multicolumn{8}{|c|}{ Cronbach's alpha if item deleted } \\
\hline Total & .78 & .77 & .75 & .74 & .76 & .73 & \\
\hline $\mathrm{MCl}$ & .60 & .62 & .59 & .55 & .56 & .57 & \\
\hline Mild dementia & .69 & .70 & .70 & .69 & .67 & .65 & \\
\hline Moderate dementia & .72 & .72 & .70 & .66 & .73 & .66 & \\
\hline
\end{tabular}

$N=443 ; \mathrm{n}(\mathrm{MCl})=91 ; \mathrm{n}($ mild dementia $)=186 ; \mathrm{n}($ moderate dementia $)=166$

MCl: MMSE score 24-30; mild dementia: MMSE score 18-23; moderate dementia: MMSE score 10-17

Mobility -.00, Self-Care -.23, Usual Activities -.20, Pain/Discomfort .15, Anxiety/Depression .01. The correlation with the Social Behavior scale from the NOSGER was -.11. The correlations between the ETAM total score and the scores from these instruments are presented in Table 3 in detail.

\section{Criterion-related validity}

We computed a one-way ANOVA to test the hypothesis that participants would receive different ETAM scores depending on their care level. The independent variable care level had three factor levels: no care level, care level 1 , and care level $2+$. We combined participants with care levels 2 and 3 because there were only four participants with care level 3 . The results showed that the ETAM scores differed significantly from each other depending on the participants' nursing care needs, $F(2,440)=$ 8.660, $p<.001$, as shown in Table 4. Hochberg's GT2 post hoc test showed that there were significant differences in ETAM scores between participants with no care level and care level $2+(\mathrm{p}<.001)$ and participants with care level 1 and care level $2+(p=.015)$. Participants with

Table 3 Discriminant validity

\begin{tabular}{lllll}
\hline & Total & MCl & Mild dementia & Moderate dementia \\
\hline MMSE & $.59^{* *}$ & .20 & $.24^{* *}$ & $.40^{* *}$ \\
Social Behavior (NOSGER) & $-.11^{*}$ & .03 & -.04 & -.01 \\
EQ-5D items & & & & -.07 \\
Mobility & -.01 & .12 & -.08 & $-.18^{*}$ \\
Self-Care & $-.23^{* *}$ & -.10 & -.20 & $-.18^{*}$ \\
Usual Activities & $-.20^{* *}$ & -.14 & .05 & -.08 \\
Pain/Discomfort & $.15^{* *}$ & .17 & .03 & .05 \\
Anxiety/Depression & .01 & .03 & & -.03 \\
\hline
\end{tabular}
${ }^{*} p<.05 ;{ }^{* *} p<.01$ 
Table 4 Criterion-related validity

\begin{tabular}{llllcc}
\hline & Number & M & SD & \multicolumn{2}{c}{ p, Hochberg's GT2 post hoc test } \\
\cline { 5 - 6 } & & & & Care level 1 & Care level 2+ \\
\hline No care level & 71 & 19.70 & 6.18 & .11 & $<.001$ \\
Care level 1 & 232 & 17.69 & 7.08 & & $<.05$ \\
Care level 2+ & 140 & 15.55 & 7.57 & & \\
\hline
\end{tabular}

no care level and care level 1 did not differ significantly in their ETAM scores $(p=.11)$.

\section{Sensitivity to change: Subgroup analysis of the control group}

In a regression analysis, when predicting the ETAM score at $\mathrm{t} 1$, we found that the ETAM score at $\mathrm{t} 0(\mathrm{~b}=0.83$, $\mathrm{p}<.001)$ and the change in MMSE from t0 to $\mathrm{t} 1 \mathrm{~b}=$ $-0.37, \mathrm{p}<.001)$ were significant predictors; see Table 5. $\mathrm{R}^{2}$ was 0.65 . This supports our hypothesis that when a person's MMSE score had declined after six months, the person also achieved fewer points on the ETAM after six months.

\section{Discussion}

In this study, we examined the reliability and validity of the ETAM and confirmed that the ETAM can be used not only with people with MCI and mild dementia but also with people with moderate dementia. We showed that ETAM scores differed between the level of cognitive impairment with people with MCI achieving the best results, people with mild dementia second best, and people with moderate dementia the worst. In addition, we confirmed that the ETAM is able to detect change over time. Also, a confirmatory factor analysis supported the postulated single factor structure of IADLs.

The present study supports the application of the ETAM for persons with MCI or mild dementia. In addition, the ETAM can also be recommended for assessing the subgroup of persons with moderate dementia. This is meaningful because functional assessment becomes more important when the degree of cognitive impairment increases [5]. Our analyses showed that persons with MCI achieved the best results, persons with mild dementia scored on average five points lower, and persons with moderate dementia scored another six points lower. Thus, these results show that as the dementing disease progresses, participants find it increasingly difficult to carry out the IADL-oriented tasks of the ETAM, thus providing support for the ETAM's reliability and validity.

Further support for the validity of the ETAM was provided by care level, which is primarily related to BADL capacities. We found that participants who had not yet qualified for a care level achieved the most points (i.e. they showed a better performance on the ETAM), and with a higher care level, participants achieved fewer points on the ETAM. Persons with no care level and persons with care level 1 did not show significantly different ETAM scores, which might be due to the different sample sizes that were used or the fact that care level is more strongly related to BADL capacities than to IADL capacities. This finding is especially interesting because care level is an external criterion that was rated by independent testers who were not involved in the study.

We were able to confirm the discriminant validity of the ETAM as predicted in our hypotheses (moderate overall correlation with the MMSE; low correlations with all other tests). Whereas the ETAM scores of people with MCI were barely correlated with the MMSE, the correlation increased when we analyzed the subgroup of persons with mild or moderate dementia. This finding is consistent with Giebel et al.'s [46] results in suggesting that with the progression of the dementing disease, cognition is increasingly affected, and people have more trouble mastering IADLs. Further support for the association between cognitive levels and functional abilities such as IADLs was found, for example, by Njegovan [47], who showed that progressive cognitive decline is associated with a specific pattern of loss of functional tasks. All in all, these findings appear to suggest that activities of daily living and cognitive tasks are increasingly associated as cognitive impairment progresses. This means that the relationship between IADL capacities and performance on cognitive tasks increases as cognitive impairment progresses. A similar yet weaker pattern was found for the correlation between the ETAM and the Self-Care item from the EQ-5D, which can be applied to assess BADLs to a certain extent. Again, as the dementing condition progressed, the correlation with the ETAM increased.

In addition, we used the five EQ-5D items to compute correlation coefficients with dimensions such as pain, anxiety, etc. Aside from Self-Care, we found no meaningful correlation or pattern of correlations across the three subgroups of participants with $\mathrm{MCI}$, mild dementia, or

Table 5 Sensitivity to Change: Subgroup Analysis of the Control Group: Regression Analysis

\begin{tabular}{|c|c|c|c|c|c|}
\hline & \multirow{2}{*}{$\begin{array}{l}\text { Estimate } \\
\text { (b) }\end{array}$} & \multirow[t]{2}{*}{ SE } & \multirow[t]{2}{*}{$p$} & \multicolumn{2}{|l|}{$95 \% \mathrm{Cl}$} \\
\hline & & & & lower limit & upper limit \\
\hline ETAM score at t0 & 0.83 & 0.05 & $<.001$ & .721 & .937 \\
\hline MMSE change score t0-t1 & -0.37 & 0.10 & $<.001$ & -.566 & -.181 \\
\hline
\end{tabular}


moderate dementia, thus providing support for the discriminant validity of the ETAM.

Another important relationship between the ETAM and the MMSE concerns sensitivity to change. For this purpose, we analyzed whether the ETAM was sensitive to other (cognitive) changes over a period of six months. We found that the change in MMSE over a period of six months turned out to be a significant predictor of the ETAM score after six months: When a person's MMSE score had declined after six months, the person also achieved fewer points on the ETAM after six months. This is an important aspect of validity and it demonstrates that the ETAM is able to measure change over time. Thus, we recommend its use in intervention studies.

Similar to the first validation study, the item "phone call" turned out to be the most difficult item by far. The authors of the first validation study argued that how a person handles the phone is an important and sensitive indicator of incipient dementia processes [22]. The item "traffic situations" was the second most difficult item. Apart from these findings, there were some differences in the order of items in comparison with the first validation study. This was most likely due to a smaller sample size in the previous study as well as less variation (the difficulties of the remaining four items ranged only from .47 to .67). In the current study, there was a consistent pattern of difficulty indices with one small exception. For the MCI subgroup, "pill organizer" and "alarm clock" were the easiest items (both .83), and "making tea" was the third easiest item (.77). Because it is common practice to arrange the items on a test in order of increasing difficulty, we propose that the order of the ETAM items be rearranged and adjusted to reflect the difficulties found in the current study. Specifically, we suggest the following order when carrying out the ETAM: 1) "making tea," 2) "alarm clock," 3) "pill organizer," 4) "finances," 5) "traffic situations," 6) "phone call." When the items are administered in this order, the participant is encouraged to continue the test, and this will also ensure that weaker candidates will not become discouraged.

\section{Limitations}

Some limitations of the current study should be mentioned. Because the lack of high-quality performance-based assessments for measuring IADL capabilities was the reason we developed the ETAM, we cannot provide convergent validity with other instruments that measure IADL capacities. To date, there is no gold standard for measuring IADL capacities especially by means of a performance-based assessment. Existing performance-based assessments are very time-consuming, taking up to $1.5 \mathrm{~h}$ [48] in only very small groups [24], or they seem to measure cognition rather than
IADL functioning [49] (for an overview, see [22]). Because the ETAM already showed acceptable convergent validity with the informant-based Bayer Activities of Daily Living Scale [50] in the first validation study [22], we decided to focus on discriminant validity and sensitivity to change.

In addition, one should consider that differentiating between $\mathrm{MCI}$, mild dementia, and moderate dementia can be performed only with the mean ETAM scores. This is because there is high between-subject variability on the ETAM in the three levels of cognitive impairment. Thus, ETAM scores should not be used to diagnose MCI, mild dementia, or moderate dementia.

Another limitation of the present study was that we used the NOSGER subscale Social Behavior and the EQ-5D items to analyze discriminant validity. However, a measure of mood would have been desirable because depressive mood is associated with a decline in cognitive abilities.

\section{Future research perspectives}

In our study, the categorization of MCI, mild dementia, and moderate dementia was solely based on the cognitive tests of the MMSE and the MoCA, which can be influenced by age and education [51, 52]. Thereby, we defined cognitive impairment psychometrically and assessed clinical symptoms. In practice, the MMSE is one of the most commonly used screening tools for cognitive impairment [32], and our analyses also showed that this categorization was successful. For a more accurate categorization for persons with $\mathrm{MCI}$ and different stages of dementia, future studies could focus on the use of other instruments besides the MMSE as well (e.g. the Consortium to Establish a Registry for Alzheimer's Disease [CERAD], neuroimaging, and biomarkers). Especially in the preclinical and prodromal stages (MCI) of Alzheimer's disease, biomarker assessments are very informative [5].

\section{Conclusions}

There is further evidence for the ETAM as a feasible, reliable, and valid instrument for the measurement of IADL capacities in persons with MCI or mild dementia. In addition, the ETAM can be recommended for the assessment of the IADL capacities of persons with moderate dementia. It shows good discriminant validities with other measures (e.g. Social Behavior, Mobility, Pain/Discomfort, and Anxiety/Depression). The ETAM is sensitive to change, and thus, we recommend its use for intervention studies.

\section{Abbreviations}

ADLs: Activities of daily living; BADLs: Basic activities of daily living; CATI: Computer-assisted telephone interview; CFA: Confirmatory factor analysis; CFI: Comparative fit index; Cl: Confidence interval; DAFA: Direct assessment of functional abilities; DAFS: Direct assessment of functional 
status; E-ADL: Erlangen test for activities of daily living; EQ-5D: EuroQol five dimensions questionnaire; ETAM: Erlangen test of activities of daily living in persons with mild dementia or mild cognitive impairment; FLSA: Functional living skills assessment; IADLs: Instrumental activities of daily living; ICF: International classification of functioning, disability, and health; MCl: Mild cognitive impairment; MMSE: Mini-mental status examination; MoCA: Montreal cognitive assessment: NOSGER: Nurses' observation scale for geriatric patients; RMSEA: Root mean square error of approximation; SD: Standard deviation; TLI: Tucker-Lewis index; WHO: World Health Organization

\section{Acknowledgments}

We acknowledge receiving funding for the study from the German National Association of the Statutory Health Insurance and Long-Term Care Insurance Funds (GKV-Spitzenverband) (Germany) and the Bavarian State Ministry of Health and Care (Germany). We would like to thank the Friedrich-AlexanderUniversität Erlangen-Nürnberg within the funding program Open Access Publishing. We would also like to thank our language editor, Dr. Jane Zagorski. The present work was conducted in partial fulfillment of the requirements for obtaining the degree "Dr. rer. biol. hum." by Stephanie Book.

\section{Ethics approval and consent to participate}

All procedures were approved by the Friedrich-Alexander-Universität of Erlangen-Nürnberg Ethics Committee (Re.-No. 170_14 B). Participation was voluntary, and subjects were free to leave the study at any time. Written informed consent was obtained from all participants.

\section{Availability of data and materials}

The datasets used and/or analyzed during the current study are available from the corresponding author on reasonable request.

\section{Authors' contributions}

SB drafted the manuscript, performed the data analysis, and developed the structure of the paper. MS provided important information about data analysis and interpretation. $\mathrm{KL}$ designed and supervised the study. SM provided important suggestions about the statistical analyses. EG designed the study, supervised the study design and data analysis, and drafted parts of the manuscript. All authors have read and approved the final version of the manuscript.

\section{Consent for publication}

Not applicable.

\section{Competing interests}

The authors declare that they have no competing interests.

\section{Publisher's Note}

Springer Nature remains neutral with regard to jurisdictional claims in published maps and institutional affiliations.

\section{Author details}

${ }^{1}$ Center for Health Services Research in Medicine, Department of Psychiatry and Psychotherapy, Friedrich-Alexander Universität Erlangen-Nürnberg, Schwabachanlage 6, 91054 Erlangen, Germany. ${ }^{2}$ Institute of Psychology, Friedrich-Alexander-Universität Erlangen-Nürnberg, Nägelsbachstr. 49c, 91052 Erlangen, Germany. ${ }^{3}$ Institute of Medical Informatics, Biometry, and Epidemiology, Friedrich-Alexander-Universität Erlangen-Nürnberg, Waldstraße 6, 91054 Erlangen, Germany.

Received: 29 May 2017 Accepted: 17 September 2018

Published online: 24 September 2018

\section{References}

1. Lawton M, Brody E. Assessment of older people: self-maintaining and instrumental activities of daily living. Gerontologist. 1969;9:179-86.

2. World Health Organization. Dementia: a public health priority. Geneva: World Health Organization; 2012.

3. Dubois B, Feldman HH, Jacova C, Dekosky ST, Barberger-Gateau P, Cummings J, Delacourte A, Galasko D, Gauthier S, Jicha G, et al. Research criteria for the diagnosis of Alzheimer's disease: revising the NINCDS-ADRDA criteria. Lancet Neurol. 2007;6(8):734-46.
4. Sperling RA, Aisen PS, Beckett LA, Bennett DA, Craft S, Fagan AM, Iwatsubo $T$, Jack CR Jr, Kaye J, Montine TJ, et al. Toward defining the preclinical stages of Alzheimer's disease: recommendations from the National Institute on Aging-Alzheimer's Association workgroups on diagnostic guidelines for Alzheimer's disease. Alzheimers Dement. 2011;7(3):280-92.

5. Ritchie CW, Russ TC, Banerjee S, Barber B, Boaden A, Fox NC, Holmes C, Isaacs JD, Leroi I, Lovestone $\mathrm{S}$, et al. The Edinburgh consensus: preparing for the advent of disease-modifying therapies for Alzheimer's disease. Alzheimers Res Ther. 2017;9(1):85.

6. Sikkes SA, Rotrou J. A qualitative review of instrumental activities of daily living in dementia: what's cooking? Neurodegenerative Disease Management. 2014;4(5):393-400.

7. Farias ST, Mungas D, Reed BR, Harvey D, Cahn-Weiner D, Decarli C. MCl is associated with deficits in everyday functioning. Alzheimer Dis Assoc Disord. 2006;20(4):217-23

8. Nygard L. Instrumental activities of daily living: a stepping-stone towards Alzheimer's disease diagnosis in subjects with mild cognitive impairment? Acta Neurol Scand Suppl. 2003;179:42-6.

9. Perneczky R, Pohl C, Sorg C, Hartmann J, Komossa K, Alexopoulos P, Wagenpfeil S, Kurz A. Complex activities of daily living in mild cognitive impairment: conceptual and diagnostic issues. Age Ageing. 2006;35(3): 240-5.

10. Perneczky R, Pohl C, Sorg C, Hartmann J, Tosic N, Grimmer T, Heitele S, Kurz A. Impairment of activities of daily living requiring memory or complex reasoning as part of the $\mathrm{MCl}$ syndrome. Int J Geriatr Psychiatry. 2006;21(2): 158-62.

11. Overdorp EJ, Kessels RP, Claassen JA, Oosterman JM. The combined effect of neuropsychological and Neuropathological deficits on instrumental activities of daily living in older adults: a systematic review. Neuropsychol Rev. 2016:26(1):92-106.

12. Boyle PA, Cohen RA, Paul R, Moser D, Gordon N. Cognitive and motor impairments predict functional declines in patients with vascular dementia. Int. J. Geriatr. Psychiatry. 2002;17(2):164-9.

13. Peres K, Helmer C, Amieva H, Orgogozo JM, Rouch I, Dartigues JF, Barberger-Gateau P. Natural history of decline in instrumental activities of daily living performance over the 10 years preceding the clinical diagnosis of dementia: a prospective population-based study. J Am Geriatr Soc. 2008; 56(1):37-44.

14. Petersen RC. Mild cognitive impairment as a diagnostic entity. J Intern Med. 2004;256(3):183-94.

15. Palmer K, Backman L, Winblad B, Fratiglioni L. Mild cognitive impairment in the general population: occurrence and progression to Alzheimer disease. J Am Geriatr Soc. 2008;16(7):603-11.

16. Winblad B, Palmer K, Kivipelto M, Jelic V, Fratiglioni L, Wahlund LO, Nordberg A, Backman L, Albert M, Almkvist O, et al. Mild cognitive impairment-beyond controversies, towards a consensus: report of the international working group on mild cognitive impairment. J Intern Med. 2004;256(3):240-6

17. Jekel K, Damian M, Wattmo C, Hausner L, Bullock R, Connelly PJ, Dubois B, Eriksdotter M, Ewers M, Graessel E, et al. Mild cognitive impairment and deficits in instrumental activities of daily living: a systematic review. Alzheimers Res Ther. 2015;7(1):17.

18. Kim KR, Lee KS, Cheong HK, Eom JS, Oh BH, Hong CH. Characteristic profiles of instrumental activities of daily living in different subtypes of mild cognitive impairment. Dement Geriatr Cogn Disord. 2009;27(3): 278-85.

19. Sikkes SAM, de Lange-de Klerk ESM, Pijnenburg YAL, Scheltens P, Uidehaag BMJ. A systematic review of instrumental activities of daily living scales in dementia: room for improvement. J Neurol Neurosurg Psychiatry. 2009;80:7-12.

20. Graessel E, Viegas R, Stemmer R, Küchly B, Kornhuber J, Donath C. The Erlangen test of activities of daily living: first results on reliability and validity of a short performance test to measure fundamental activities of daily living in dementia patients. International Psychogeriatrics / IPA. 2009;21(1):103-12.

21. Luttenberger K, Schmiedeberg A, Graessel E. Activities of daily living in dementia: revalidation of the E-ADL test and suggestions for further development. BMC Psychiatry. 2012;12(1):208.

22. Luttenberger K, Reppermund S, Schmiedeberg-Sohn A, Book S, Graessel E. Validation of the Erlangen test of activities of daily living in persons with mild dementia or mild cognitive impairment (ETAM). BMC Geriatr. 2016;16(1):12.

23. Farina E, Fioravanti $R$, Pignatti $R$, Alberoni M, Mantovani F, Manzoni G, Chiacari L, Imbornone E, Villanelli F, Nemni R. Functional living skills 
assessment: a standardized measure of high-order activities of daily living in patients with dementia. Eur J Phys Rehabil Med. 2010;46:73-80.

24. Karagiozis H, Gray S, Sacco J, Shapiro M, Kawas C. The direct assessment of functional abilities (DAFA): a comparison to an indirect measure of instrumental activities of daily living. Gerontologist. 1998;38:113-21.

25. McDougall G, Becker $H$, Vaughan P, Acee TW, Delville CL. The revised direct assessment of functional status of independent older adults. Gerontologist. 2010;50:363-70.

26. World Health Organization: International Classification of Functioning, Disability and Health (ICF). Geneva: World Health Organization; 2001.

27. Graessel E, Stemmer R, Eichenseer B, Pickel S, Donath C, Kornhuber J, Luttenberger K. Non-pharmacological, multicomponent group therapy in patients with degenerative dementia: a 12-month randomised, controlled trial. BMC Med. 2011;9(1):129.

28. Straubmeier M, Behrndt EM, Seidl H, Oezbe D, Luttenberger K, Graessel E: Effekte einer nichtpharmakologischen Mehrkomponententherapie in Tagespflege-Einrichtungen auf Menschen mit kognitiven Einschränkungen Ergebnisse der randomisierten kontrollierten "German Day-Care Study". Deutsches Ärzteblatt (accepted).

29. World Health Organization. The international classification of functioning, disability and health: ICF. Geneva: World Health Organization; 2001.

30. Muò R, Schindler A, Vernero I, Schindler O, Ferrario E, Frisoni GB. Alzheimer's disease-associated disability: an ICF approach. Disabil Rehabil. 2005;27:1405-13.

31. Folstein M, Folstein S, Mc Hugh P. "Mini-mental state": a practical method for grading the cognitive state of patients for the clinician. J Psychiatr Res. 1975;12(3):189-98.

32. Arevalo-Rodriguez I, Smailagic N, Roque IFM, Ciapponi A, Sanchez-Perez E, Giannakou A, Pedraza OL, Bonfill Cosp X, Cullum S. Mini-mental state examination (MMSE) for the detection of Alzheimer's disease and other dementias in people with mild cognitive impairment (MCl). Cochrane Database Syst Rev. 2015;3:CD010783.

33. Tombaugh TN, Mclntyre NJ. The mini-mental state examination: a comprehensive review. J Am Geriatr Soc. 1992;40(9):922-35.

34. Nasreddine ZS, Phillips NA, Bedirian V, Charbonneau S, Whitehead V, Collin I, Cummings JL, Chertkow H. The Montreal cognitive assessment, MoCA: a brief screening tool for mild cognitive impairment. J Am Geriatr Soc. 2005; 53(4):695-9.

35. Freitas S, Simoes MR, Alves L, Santana I. Montreal cognitive assessment: validation study for mild cognitive impairment and Alzheimer disease. Alzheimer Dis Assoc Disord. 2013;27(1):37-43.

36. Larner AJ. Screening utility of the Montreal cognitive assessment (MoCA): in place of - or as well as - the MMSE? Int Psychogeriatr. 2012;24(3):391-6.

37. Dong Y, Lee WY, Basri NA, Collinson SL, Merchant RA, Venketasubramanian $\mathrm{N}$, Chen $\mathrm{CL}$. The Montreal cognitive assessment is superior to the minimental state examination in detecting patients at higher risk of dementia. Int Psychogeriatr. 2012;24(11):1749-55.

38. The EuroQol Group. EuroQol - a new facility for the measurement of healthrelated quality of life. Health Policy. 1990;16:199-208.

39. Spiegel R, Brunner C, Ermini-Fünfschilling D, Monsch A, Notter M, Puxty J, Tremmel L. A new behavioral assessment scale for geriatric out- and inpatients: the NOSGER (nurses' observation scale for geriatric patients). J Am Geriatr Soc. 1991;39(4):339-47.

40. Ihl R, Frolich L, Dierks T, Martin EM, Maurer K. Differential validity of psychometric tests in dementia of the Alzheimer type. Psychiatry Res. 1992; 44(2):93-106.

41. Peterson RA. A meta-analysis of Cronbach's coefficient alpha. J Consum Res. 1994;21(2):381-91

42. Fisseni HJ. Lehrbuch der psychologischen Diagnostik. Göttingen: Hogrefe; 1997.

43. Bortz J, Döring N. Forschungsmethoden und Evaluation: für Human- und Sozialwissenschaftler. Berlin, Heidelberg: Springer; 2006.

44. Brown TA. Confirmatory factor analysis for applied research. New York: The Guilford Press; 2006.

45. Schreiber JB, Nora A, Stage FK, Barlow EA, King J. Reporting structural equation modeling and confirmatory factor analysis results: a review. J Educ Res. 2006;99(6):323-37.

46. Giebel CM, Challis DJ, Montaldi D. A revised interview for deterioration in daily living activities in dementia reveals the relationship between social activities and well-being. Dementia (London). 2014:1-14.
47. Giebel CM, Challis, DJ, Montaldi D: A revised interview for deterioration in daily living activities in dementia reveals the relationship between social activities and well-being. Dementia 2016, 15(5):1068-81.

48. Moore DJ, Palmer BW, Patterson TL, Jeste DV. A review of performance-based measures of functional living skills. J Psychiatr Res. 2007;41(1-2):97-118.

49. Cullum CM, Saine K, Chan L, Martin-Cook K, Gray K, Weiner M. Performancebased instrument to assess functional capacity in dementia: the Texas functional living scale. Neuropsychiatry Neuropsychol Behav Neurol. 2001; 14:103-8.

50. Hindmarch I, Lehfeld $H$, Jongh $P$, Erzigkeit $H$. The Bayer activities of daily living scale (B-ADL). Dement Geriatr Cogn Disord. 1998;9:20-6.

51. Crum RM, Anthony JC, Bassett SS, Folstein MF. Population-based norms for the mini-mental state examination by age and educational level. Jama. 1993;269(18):2386-91.

52. Freitas S, Simoes MR, Alves L, Santana I. Montreal cognitive assessment: influence of sociodemographic and health variables. Arch Clin Neuropsychol. 2012;27(2):165-75

\section{Ready to submit your research? Choose BMC and benefit from:}

- fast, convenient online submission

- thorough peer review by experienced researchers in your field

- rapid publication on acceptance

- support for research data, including large and complex data types

- gold Open Access which fosters wider collaboration and increased citations

- maximum visibility for your research: over $100 \mathrm{M}$ website views per year

At BMC, research is always in progress.

Learn more biomedcentral.com/submissions 